\title{
Susanne Söderberg
}

\section{Das amerikanische Empire und die "ausgeschlossenen Staaten" Das Millenium Challenge Account - eine „preemptive" Entwicklungspolitik ${ }^{1}$}

America's cause is the cause of all mankind. Benjamin Franklin, zitiert nach Kagan 2003

Im März 2002 schlug Präsident George W. Bush ein neues globales Entwicklungshilfeprogramm vor: Das Millenium Challenge Account (MCA). ${ }^{2}$ Im Rahmen dieses Programms wird die US-Regierung in den kommenden drei Jahren die Kernbereiche ihrer Entwicklungshilfe ausbauen. Bereits laufende Kredite an Länder des Südens, sollen dabei durch „Fördergelder“ (grants) ersetzt werden. Diese Gelder sollen an Regierungen vergeben werden, „who rule justly, invest in their people, and encourage economic freedom" (Daalder et. al. 2002). Die Vergabe von Finanzmitteln wird von 16 Kriterien abhängig gemacht, welche die Empfängerländer einhalten müssen, um sich für die Entwicklungshilfe zu qualifizieren - angefangen bei der Einhaltung bürgerlicher Freiheitsrechte über die Dauer der Genehmigung eines Geschäftsbetriebes bis hin zur Handelspolitik. Das MCA spiegelt den Wandel des amerikanischen Imperialismus wider, wie er nach den tragischen Ereignissen vom 11. September 2001 deutlich wird. Sichtbar wird dieser Wandel schon daran, dass der amerikanische Staat in zunehmendem Maße die Position vertritt, USamerikanische Werte und Regeln hätten als weltweit erstrebenswert zu gelten. Offensiv wurde dies sowohl im Project for the New American Century (vgl. http://www.newamericancentury. org/) als auch in der American National Security Strategy (NSS) aus dem Jahr 2002 formuliert.

Im Hinblick auf die Beziehungen zwischen der US-Regierung und der Zielgruppe des MCA, den weltweit 79 ärmsten Ländern, bei denen anscheinend unterstellt wird, dass gescheiterte Staaten (failed states) besonders häufig vertreten sind, steht die NSS für mindestens zwei wichtige Wendepunkte. ${ }^{3}$ Erstens

1 Die im Rahmen dieses Artikels vorgetragenen Argumente entwickle ich ausführlich in: Global Governance and American Empire (Söderberg im Erscheinen).

2 Am 18. Juni 2003 stimmten das Senate Foreign Relations Committee und das House International Relations Committee der Einrichtung des MCA zu.

3 Viele Kommentatoren haben eine auffällige Ähnlichkeit zwischen der NSS der zweiten Bush

PROKLA. Zeitschrift für kritische Sozialwissenschaft, Heft 135, 34. Jg., 2004, Nr. 2, 299-319 
dulden die USA keine moderaten oder gar kritischen Positionen gegenüber ihrem „Krieg gegen den Terrorismus“. Es gibt nur die Möglichkeit für oder gegen die Vereinigten Staaten zu sein. Diese Haltung, die mit dem Begriff der „preemptiven Außenpolitik“ bezeichnet wird, stellt darauf ab, dass die USA ihre militärische Stärke auch ohne Angriff permanent demonstrieren und zur Vorbeugung terroristischer Akte einsetzen sollten. ${ }^{4}$ Zweitens sind die USA der Überzeugung, dass nach dem Ende des Kalten Kriegs eine gerechtere und friedlichere Staatenwelt dadurch zu erreichen ist, dass amerikanische Werte und Regeln in den Länder des Südens kodifiziert werden (Dodge 2002). Obwohl diese Politik für die ärmsten Regionen der Welt von großer Bedeutung ist, und auch Licht auf das sich neu formierende amerikanische Empire in der Welt nach dem 11. September wirft, hat es bisher keine systematischen Versuche einer kritischen Einschätzung dieser neuen entwicklungspolitischen Strategie gegeben. Warum wurde das MCA eingerichtet? Was hat die USA, die gemessen an der Größe ihrer Volkswirtschaft zu den weltweit geizigsten Geberländern gehört, dazu bewogen, neue Formen der Auslandshilfe für die ärmsten Länder der Welt bereit zu stellen? Und welche Interessen werden durch das MCA bedient?

Diesen Fragen soll hier nachgegangen werden, indem das MCA als Bestandteil einer historisch-spezifischen imperialistischen Politik der USA diskutiert wird. ${ }^{5}$ Wie Leo Panitch und Sam Gindin richtig feststellen, liegen die schwerwiegendsten Probleme für den US-Imperialismus heute bei den sogenannten „ausgeschlossenen Staaten“ (excluded states) bzw. dem nicht integrierbaren Spalt (non-integrating gap). Beide Begriffe beschreiben diejenigen Länder, die nicht unmittelbar in den globalen Kapitalismus integriert sind, so dass sie weder durch externe ökonomische Kräfte noch durch internationale Institutionen effektiv restrukturiert werden könnten (siehe Amin 1999). ${ }^{6}$ Die Ereignisse vom 11. September haben den non-integrating gap zu einem zentralen Aspekt amerikanischer Außenpolitik werden lassen. Im vorliegenden Text werden die terroristischen Angriffe jedoch weder zum Ausgangspunkt der Analyse gemacht, noch als eine Ursache für das MCA angesehen. Vielmehr wird hier argumentiert, dass die Form des MCA zwar neu ist, der Inhalt jedoch früheren Entwicklungshilfeprogrammen gleicht. Die Form des MCA ist insofern neu, weil sie eine unverhohlene Artikulation US-amerikanischer Interessen gegenüber den ausgeschlossenen Staaten des Südens - so wie sie in der neuen Phase

Regierung und dem Entwurf des Defence Planning Guidance Dokuments von 1992 durch Paul Wolfowitz (Deputy Secretary of Defence) festgestellt. Vgl. z.B. PBS Frontline: http://www.pbs.org/wgbh/pages/frontline/shows/iraq/etc/wolf.html

4 Der Begriff ,Terrorismus ist höchst umkämpft. Seine Bedeutung hängt vor allem davon ab, wer ihn definiert. Siehe Willetts (1999: 297).

5 Siehe die exzellenten Essays im Socialist Register 2004, wie etwa die von Panitch/Gindin, Albo oder Harvey.

6 Der Begriff non-integrating gap ist zitiert nach dem Beratungspapier eines Professors des US Naval War College für das Secretary of Defense (in: Panitch/Gindin 2004: 31). 
imperialistischer US-Politik definiert werden - darstellt. Insofern spreche ich hier von einer „preemptiven Entwicklungspolitik“. Während im Rahmen der Strategie der Konditionalität die Vergabe von IWF- und Weltbank-Krediten an Bedingungen geknüpft wurde, welche die Empfängerländer nach Bewilligung der Mittel einhalten mussten, funktioniert preemptive Entwicklungspolitik genau anders herum: Die zugesagten Mittel werden erst ausgezahlt, wenn alle Forderungen des Geldgebers erfüllt sind, was durch quantitative Messverfahren kontrolliert wird. Bei näherer Betrachtung zeigt sich jedoch, dass die an das MCA geknüpften Politikvorstellungen die gleichen Ziele und Interessen beinhalten, wie sie in den vergangenen zwei Jahrzehnten auch im Rahmen des Washington-Konsensus verfolgt wurden: Wachstum und Wohlstand hängen demnach vom Willen und der Fähigkeit der betroffenen Länder $a b$, eine liberale Wirtschaftspolitik und die Geltung des bürgerlichen Rechts durchzusetzen (Privateigentum, Kommodifizierung und Privatisierung von Land, etc.).

Im ersten Teil des Aufsatzes sollen die grundlegenden Machtbeziehungen und Widersprüche herausgearbeitet werden, aus denen das MCA hervorgegangen ist. Dazu ist es notwendig, das MCA in einem größeren Kontext zu diskutieren, soll doch über die einfache Annahme hinausgegangen werden, dass die Einführung und öffentliche Rechtfertigung des MCA ausschließlich auf die tragischen Vorfälle vom 11. September zurückzuführen sind. Gerade der Rekurs auf den 11. September legitimiert nicht nur die zunehmend repressive Politik gegenüber ausgewählten Ländern des Südens, was unter anderem auch den Trend zur Privatisierung und Militarisierung der Entwicklungspolitik einschließt. Er dient auch der Verdunkelung tiefer liegender Gründe für die Einführung des MCA und der Verschleierung spezieller Interessen, denen das MCA dient. Eine Auseinandersetzung damit wird dann im zweiten Teil des Textes geleistet. Hier soll gefragt werden, welche Ziele das MCA verfolgt, wie diese erreicht werden und welche Organisationen in diesen Prozess involviert sind.

\section{Vom Washington Konsens zum Post-Washington Konsens}

Vom Beginn der Schuldenkrise in den frühen 1980ern bis in die späten 1990er Jahre war die Form der offiziellen Entwicklungspolitik durch den Konsens von Washington geprägt. Dieser basierte auf der festen Überzeugung, dass politische und soziale Probleme nicht durch staatliche Interventionen, sondern primär durch marktförmige Mechanismen und die Durchsetzung bürgerlichen Rechts gelöst werden sollten. Die Prinzipien des Neoliberalismus, auf denen dieser Konsens beruht, wurden schnell zur Leitpolitik der internatonalen Finanzinstitutionen und der größten bilateralen Hilfsagentur, der United States Agency for International Development (USAID) (Fine 2001). Der Konsens von Washington operierte auf Grundlage der normativen Prämisse, dass die Kräfte des Marktes über die des Staates dominieren sollen, dass Staaten ihren politi- 
schen Gestaltungsanspruch weitgehend aufzugeben hätten, abgesehen von ihrer Aufgabe, das bürgerliche Recht zu garantieren und durchzusetzen (z.B. private Eigentumsrechte, Repatriierung von Vermögen und Kapital, usw.). Die Politikrezepte des Konsens von Washington zielten daher vor allem auf die Implementierung einer soliden Wirtschaftspolitik und marktfreundlicher Reformen in den Ländern des Südens, wie auch darauf, die betreffenden Ländern dabei zu unterstützen, ökonomische Stabilität und Prosperität zu erreichen.

Thomas Friedman, Kolumnist der New York Times und überzeugter Verfechter des Neoliberalismus, bringt die Rationalität des Konsens von Washington auf den Punkt, wenn er von der „goldenen Zwangsjacke“ der Globalisierung spricht (Friedman 1999). ${ }^{7}$ Friedman meint, die Zwangsjacke „will force contentious publics to understand the logic of globalisation is that of peace (since war would interrupt globalisation and therefore progress) and democracy (because new technologies increase individual autonomy and encourage initiative)" (Hoffman 2002: 106). Was Friedman wie auch andere Neoliberale nicht sehen, ist, dass die der globalen Kapitalakkumulation inhärenten Widersprüche unvermeidlich Unsicherheit für die Menschen produzieren. Die neoliberale Globalisierung setzt eine Priorität von „economic growth and market logics over all other goals and institutions of governance and enforces on all national polities, with varying degrees of coercion, privatisation, trade liberalization, the deregulation of capital, and the erosion of the public sector and of democratic control“ (Brodie 2003). Die Reproduktion der neoliberalen Globalisierung ist also kein reibungsloser Prozess, sondern voller Widersprüche. Wie Elmar Altvater betont, stellt die globale Kapitalakkumulation ein historisches System dar, in welchem die endlose Akkumulation von Kapital strukturell den gesamten gesellschaftlichen Produktions- und Reproduktionszusammenhang dominiert. Dies impliziert auch, dass die Institutionen und Akteure (z.B. der IWF, bilaterale Hilfswerke, Credit-rating Agenturen, private Investoren und Kreditgeber), welche den Rahmen der Globalisierung konstituieren, diejenigen belohnen, die die endlose Kapitalakkumulation durchsetzen und jene bestrafen, die dies nicht tun. Mehr noch: der Prozess der Realisierung von Profiten, der Akkumulation und der institutionellen Regulation, verleiht dem System zwar zu einem gewissen Grad Sicherheit, produziert aber gleichzeitig auf allen Ebenen des sozialen und individuellen Lebens Unsicherheiten (Altvater 2002: 75; Altvater/Mahnkopf 2002). Letzteres kann als „Sicherheit-Unsicherheits Paradoxon" der neoliberalen Globalisierung bezeichnet werden.

$\mathrm{Da}$ es sich beim Neoliberalismus um eine historisch-spezifische Form des globalen Kapitalismus handelt, ist auch er vom Sicherheit-Unsicherheits Paradoxon durchdrungen. Die transnationalen Kapitalklassen und politischen Eliten

7 Eine ausgezeichnete Kritik an Friedman findet sich auf Mark Ruperts Website The AntiFriedman Page: http://www.maxwell.syr.edu/maxpages/faculty/merupert/Anti-Friedman.htm 
innerhalb und außerhalb des amerikanischen Staates sind mit dessen Bewältigung konfrontiert. Seit dem Ende von Bretton Woods (1944-1971) hat der amerikanische Imperialismus gegenüber den Ländern des Südens versucht, dem Sicherheit-Unsicherheits Paradoxon vor allem mit ökonomischem und physischem (militärischem) Zwang zu begegnen, etwa durch Strukturanpassungsprogramme (SAPs) des IWF und militarisierte Wiederaufbauprogramme in Kriegsgebieten wie Afghanistan, Bosnien und dem Irak. Es ist genau dieses Bemühen, das Sicherheit-Unsicherheits Paradoxon zu bewältigen, das die sich wandelnde Form des amerikanischen Empires im Umgang mit den excluded states kennzeichnet. Anders gesagt: In dem Maße, in dem die USA versucht, der Bedrohung ihrer hegemonialen Position entgegen zu wirken, lässt sich auch eine Veränderung in der Form der offiziellen Entwicklungspolitik beobachten, oder, was das gleiche ist: die entwicklungspolitischen Inhalte nehmen einen zunehmend repressiven Charakter an.

Die (gut dokumentierten) Armutsraten und Einkommenspolarisierungen haben im Süden zu großer Unzufriedenheit über die neoliberalen Politikrezepte der internationalen Finanzinstitutionen geführt. Mitte der 1990er Jahre begann der wachsende Unmut ernsthaft Probleme für die Reproduktion der neoliberalen Globalisierung aufzuwerfen (UNDP 1999). Die gefährliche Kombination aus einer nachlassenden Akzeptanz für marktorientierte Restrukturierungsund Sparpakete einerseits und der Einengung nationaler wirtschafts- und sozialpolitischer Handlungsspielräume andererseits, hat in vielen Ländern des Südens zu einer Krise neoliberaler Regierungspolitik geführt. Belegt wird dies etwa durch die Tatsache, dass Zwei Drittel der Weltbevölkerung im Jahr 2003 dem Satz, ihre Länder würden „governed by the will of the people“, nicht zustimmten. ${ }^{8}$ Anders ausgedrückt hat sich die allgemein übliche Annahme, die Entfesselung des Marktes steigere den Wohlstand der Bevölkerungsmehrheit, nicht nur als falsch erwiesen, sondern diese Politik hat auch zu einem Mangel an öffentlicher Unterstützung für neoliberale Prinzipien geführt. Bisher bestand die traditionelle Antwort der offiziellen Entwicklungsagenda auf die Zerstörung der materiellen Lebensbedingungen und auf die Legitimationskrise im Süden darin, darauf zu bestehen, dass die Entwicklungsländer auch weiterhin marktorientierte Restrukturierungsmaßnahmen zu implementieren hätten: je strikter Regierungen sich der Disziplinierung durch Akteure unterwerfen, die sich vor allem an ökonomischen Rationalitäten orientieren, desto mehr Wohlstand würden sie erreichen, so die Argumentation. Mindestens drei Faktoren komplizieren diese Strategie jedoch und haben in den späten 1990ern zu einer erneuten Erfindung des Konsens von Washington geführt.

8 Dieses Ergebnis wurde beim World Economic Forums 2003 bekannt gegeben. Von Gallup International and Environics wurden Daten von 34.000 Personen aus 46 Ländern ausgewertet, www.weforum.org, zitiert in Brodie (im Erscheinen). 
Erstens wurde der wachstumsbesessene und „one-size-fits-all“ Charakter der SAPs nicht nur von der Anti-Globalisierungsbewegung bei Protesten gegen die Gipfeltreffen der internationalen Finanzinstitutionen und der Welthandelsorganisation (WTO) in Seattle, Genua, Prag, Washington usw. stark kritisiert. Kritik wurde auch von Keynesianern, von konservativen Experten und in den Think-Tanks in Washington formuliert.' Im Zuge der Finanzkrisen und der darauf folgenden IWF-geführten Bailouts (Kreditvergaben um die Zahlungsfähigkeit sicherzustellen) in den sogenannten emerging markets spitzte sich die Kontroverse sogar noch zu (siehe z.B. Söderberg 2004). Zweitens hat sich der Umfang öffentlicher Hilfsleistungen schnell verringert (Ocampo 2000: 43). In den USA, die weltweit die meisten bilateralen Finanzhilfen geben, haben die Ausgaben in diesem Bereich seit den 1960er Jahren deutlich abgenommen. Allein während der Regierungszeit Clintons sanken die für Entwicklungshilfe zur Verfügung gestellten Mittel (hierunter fallen nach einer weiten Definition: Entwicklungshilfe, humanitäre Hilfe und Wirtschaftshilfe) um 370 Millionen Dollar auf 10,7 Milliarden Dollar im Jahr 2001 (Shapiro September 2000). Drittens hat sich seit Ende des Kalten Krieges die US-amerikanische Einschätzung zentraler Sicherheitsbedrohungen gewandelt. Besonders die amerikanische Sorge um die Außenpolitik anderer Staaten, z.B. wegen der Verletzung internationaler Normen durch Terrorismus oder territoriale Aggressionen, hat sich zu einem Interesse an der Innenpolitik fremder Regime verschoben, etwa $\mathrm{zu}$ der Frage des Umgangs der betreffenden Regierungen mit der eigenen Bevölkerung (Thomas 2002: 114; UNDP 1994). Um die „Neuen Kriege“ (Kaldor 1999) zu bekämpfen, setzte die Regierung Clinton auf ein multilaterales Vorgehen, vor allem im Rahmen ihrer Global Governance Politik. ${ }^{10}$ Kurz gesagt zielte Clintons Form der Global Governance, auch bekannt als Strategie des „engagement and enlargement“, auf eine (erzwungene) Transformation der Länder der Dritten Welt (vor allem der Schurkenstaaten, der failed states und der emerging markets) in demokratische Staaten, die die Prinzipien der freien Marktwirtschaft unterstützen (Clinton 1998; Litwak 2000: 49).

Als Antwort auf die oben skizzierten Widersprüche bildete sich eine neue Form der offiziellen Entwicklungshilfe heraus. Ausgestattet mit neuen Modewörten wie etwa „Eigentumsrechte“ und soziale Inklusion, zielte diese nicht etwa auf eine Verringerung, sondern auf eine Ergänzung der Disziplinierung durch den Markt ab. Inklusion, so die Weltbank, „treats poor people as co-

9 So kritisierten z.B. die Heritage Foundation (heute einer der Schlüsselakteure des MCA) und das Cato Institute, der IWF würde das Prinzip des Risikos auf privaten Märkten ausschalten, indem er durch bailouts und Hilfspakete in die „natürliche Rationalität“ von Märkten interveniere, siehe Feulner (1998) und Johnson/Schaefer (1997).

10 Der Begriff Global Governance bleibt stets schwammig. Eine Definition, die die Politik der Regierung Clinton am ehesten trifft, findet sich im Report of the Commission on Global Governance von 1995 (Commission 1995). 
producers, with authority and control over decisions and resources devolved to the lowest appropriate level“ (World Bank, o.J.) „Eigentumsrechte“ stellen die Empfängerländer im Prozess der Auswahl des Policy-mixes her, indem sie die Verantwortung für deren Implementierung und für die Politikergebnisse übernehmen (Graham/Masson 2002). Auf der Grundlage dieser neuen Agenda - auch als Post-Washington Konsensus bezeichnet - wird das Problem der „Entwicklung“ (soll heißen der Integration der armen Länder in den Weltmarkt durch Umsetzung neoliberaler Regierungsprogramme) auf Probleme des Umgangs mit Marktdefiziten reduziert. Dies betrifft vor allem ökonomische Instabilitäten und die daraus resultierenden Effekte der Ausbreitung von Finanzkrisen in den 1990er Jahren. Ökonomische Analysen werden auf nichtökonomische Bereiche ausgedehnt, um dann aus dieser Perspektive das Soziale und das Politische $\mathrm{zu}$ bewerten und konkrete Handlungsempfehlungen $\mathrm{zu}$ formulieren (Fine 2001).

Der Wandel, den die Weltbank durchlaufen hat, betrifft nicht nur die Verschiebung ihres top-down Ansatzes und der vorwiegend ökonomischen Ausrichtung hin $\mathrm{zu}$ einer vorgeblich stärker humanitär ausgerichteten Haltung (empowering development). $\mathrm{Zu}$ beobachten ist auch eine stärkere Balancierung zwischen Produktivitätssteigerung und Armutsbekämpfung. Seit Mitte 1995 hat die Weltbank ihren Fokus von der Finanzierung von Infrastrukturprojekten im Süden hin zu Programmen zur Bekämpfung der Armut verschoben. Auch der IWF spielt eine wichtige Rolle im Kampf gegen die Armut. Die Neufokussierung drückte sich etwa in der Einrichtung der Poverty Reduction Strategy Papers (PRSP) aus (IMF 2003). Allerdings geht es dabei etwa darum, das Prinzip der Konditionalität abzuschaffen. Statt dessen kann das Programm als direkte Antwort auf die oben erwähnten Bedrohungen der neoliberalen Globalisierung betrachtet werden, die dazu dient die vom US-amerikanischen Imperialismus angeführte Nord-Süd-Dominanz auszuweiten. Tatsächlich haben die jüngsten Transformationen den Druck durch marktorientierte Wachstumspolitik nicht abgeschafft. Vielmehr legitimieren sie diese Politik, indem sie die sozialen Kosten der neoliberalen Reformen dämpfen, gleichzeitig aber die Macht der transnationalen Kapitale in den betreffenden Ländern sichern (Gill/Law 1999). Tatsächlich werden die Weltbank-Programme zur Armutsbekämpfung von einer zunehmenden Kontrolle gegenüber den Ländern des Südens begleitet. Letztendlich handelt es sich beim empowering development um den Versuch, die Werte und Normen des Neoliberalismus (z.B. Markfreiheit und bürgerliches Recht) in die sich rapide verschlechternden sozialen, politischen und ökonomischen Lebensbedingungen in den Ländern des nonintegrating gap einzubetten.

Allerdings ist die Strategie des empowering development nicht in der Lage, die oben skizzierten Widersprüche der neoliberalen Globalisierung in den ,ausgeschlossenen Staaten“ zu bewältigen. Denn sie bietet keine Perspektive, das Si- 
cherheit-Unsicherheits Paradoxon im Süden, oder auch die neuen Formen der Unsicherheit in den Vereinigten Staaten zu bewältigen.

\section{Neue Sicherheitskonzepte und ökonomische Bedrohungen vor und nach 11. September}

\section{Spekulative Blasen und preemptive Sicherheitspolitik}

In der zweiten Hälfte der 1990er Jahre, nach der tiefsten Rezessionsperiode der Nachkriegszeit, begann die US-Ökonomie wieder als weltweiter Wachstumsmotor zu fungieren. So beschleunigte sich das Wachstum des BIP, es stieg zwischen 1995 und 2000 von 3,1\% auf 4,1\% (Kotz 2003: 21). Die Hauptquelle für das amerikanische Wirtschaftswachstum waren jedoch nicht, wie dies offiziell meist behauptet wird, neoliberale Reformen und der Boom der New Economy, sondern vielmehr die spekulative Blase am Finanzmarkt (Shiller 2000). Als die spekulationsbasierte Expansion Ende August 2000 mit Pauken und Trompeten zu einem Ende kam, wurde nicht nur die Fragilität des „jobless growth“ in den Industrienationen sichtbar. Angesichts der zunehmenden Abhängigkeit der Dritten Welt von der US-Wirtschaft, verdeutlichte der plötzliche Crash in den USA auch das hohe Maß an Unsicherheit in den Ländern des Südens.

Nach dem Platzen der spekulativen Finanzblase hat die USA mit hoher Arbeitslosigkeit und Unternehmensskandalen zu kämpfen. Und obwohl die USZentralbank die Zinsen auf ein Rekordtief drückte, das es seit 1958 nicht mehr gegeben hat, wird das Wirtschaftswachstum weiterhin durch ein niedriges Investitionsniveau und ein geringes Konsumentenvertrauen gedämpft. ${ }^{11}$ Auch die steigende Verschuldung der Konsumenten und des Staates haben daran nichts geändert. ${ }^{12}$ Die Tatsache, dass die USA nicht mit den eigenen Finanzmitteln zu wirtschaften vermag, den „ausgeschlossen Staaten“ aber gleichzeitig eine strenge Finanzdisziplin auferlegt, erschüttert das Vertrauen in das amerikanische Finanzmanagement. Darüber hinaus stellt das derzeitige US-Haushaltsdefizit nach Einschätzung des IWF eines der größten Risiken für die Weltwirtschaft dar (The Economist 2002a: 12). Und dass die ständig wachsenden Haushaltslöcher mit kurzfristigen Krediten gestopft werden, stellt eine weitere Quelle der Unsicherheit für die US-Ökonomie dar (Monthly Review 2003: 26). Wie oben festgestellt wurde, führte die schlechte wirtschaftliche Situation zu einer Verschärfung des Sicherheit-Unsicherheits Paradoxon in den USA wie auch im Süden. Trotz der Rhetorik des empowering development sind auch Privatinvestitionen, die den Großteil des in die Entwicklungsländer investierten Ka-

11 Nach Angaben des US Labour Department stieg die Arbeitslosenrate im Mai auf 6,1\% und befand sich damit auf dem höchsten Niveau seit Juli 1994 (siehe auch The Economist 2003; und Financial Times 2003b).

12 Economic Snapshots, http://www.epinet.org/index.html 
pitals ausmachen, von der weltweiten Rezession betroffen (The Conference Board 2003). Eine Analyse des Rückgangs der Auslandsinvestitionen in den Süden findet sich im Bericht der Weltbank Global Development Finance 2003. Hier wird festgestellt, dass der „decline since 1997 has occurred primarily in net capital flows from the private sector, particularly in the debt component (banks and bonds). From the peak years of 1995-96, when net debt inflows from the private sector were about $\$ 135$ billion per year, they have dropped steadily, becoming net outflows in 2001 and 2002" (World Bank 2003: 7). Der Trend der Kapitalflüsse hat sich zu einer Bewegung vom Süden in den Norden verkehrt. Nach Angabe des in Washington ansässigen Center for Economic and Policy Research sind die meisten Entwicklungsländer große Kapitalexporteure, was sich unter anderem den Zahlungen von Zinsen und Profiten verdankt (Morrissey/Baker 22. März 2003). Besonders dramatisch stellt sich die Situation für die ärmsten Länder des Südens dar in Zeiten einer Rezession in den USA.

Die US-amerikanische Ökonomie produzierte in der Zeit unmittelbar nach der spekulativen Blase zwei Probleme für die Reproduktion der neoliberalen Globalisierung. Angesichts der sich verschlechternden ökonomischen und sozialen Bedingungen in den USA und in den Ländern des Südens bestand das unmittelbare Problem für die herrschenden Klassen darin, die neoliberale Globalisierung weiterhin zu legitimieren. Wie David Kotz es ausdrückt: „One hindrance to the US ruling class agenda of creating a neoliberal world system has been the glaring absence of convincing evidence that neoliberal restructuring produces the benefits claimed by its promoters" (Kotz 2003: 16). Dies zeigt sich beispielsweise daran, dass Präsident Bush in der Woche vor dem 11. September 2001 in den Meinungsumfragen auf dem bis dato niedrigsten Niveau rangierte. Nur 50\% der Befragten gaben ihm zum damaligen Zeitpunkt eine positive Bewertung (Rampton/Stauber 2003). Darüber hinaus musste die USA angesichts der schlechten wirtschaftlichen Lage ihre Aktivitäten auf dem industriellen und dem Finanzsektor über den Binnenmarkt hinaus ausdehnen (David Harvey spricht in diesem Zusammenhang von einer „Akkumulation durch Enteignung"). Dazu war die USA jedoch auf die Kooperation der Regierungen im Süden angewiesen. Galt es doch, in den betreffenden Ländern die für die Expansion notwendigen politischen Programme zu entwickeln und zu implementieren, die Interessen der transnationalen Kapitale dort zu unterstützen und zu schützen, den Arbeitsmarkt zu disziplinieren und niedrige Umwelt- und Steuerstandards durchzusetzen. Angesichts der schlechten Lage der amerikanischen Wirtschaft war die Durchsetzung des Wirtschaftsliberalismus und der Geltung bürgerlichen Rechts in den ausgeschlossenen Staaten zu einem zentralen Aspekt der offiziellen Entwicklungspolitik geworden.

Generell gelten die Vorfälle vom 11. September 2001 als Hauptursache für die Erneuerung des US-amerikanischen Unilateralismus. Hier soll jedoch argumentiert werden, dass die Intensivierung des Imperialismus eher auf eine 
Kombination verschiedener Faktoren zurückzuführen ist: auf sich zuspitzende Legitimationsprobleme in Verbindung mit der turbulenten amerikanischen und globalen Wirtschaftslage, auf die Wahl einer neokonservativen USRegierung und das Unvermögen des Weißen Hauses, eine klare Strategie zur Bewältigung der wirtschaftlichen Krise auszuarbeiten. Neokonservative Experten, wie zum Beispiel Robert Kagan, haben darauf hingewiesen, dass Amerikas Rückkehr zur Machtpolitik in wichtigen geopolitischen Bereichen wie etwa der Wirtschafts- und Militärpolitik, bereits auf die Zeit vor den Angriffen auf das World Trade Center und das Pentagon zu datieren ist. Erinnern wir uns, dass sich die Bush Regierung schon vor dem 11. September offensiv in Richtung eines unilateralen Kurses bewegt hatte. Als Belege dafür können der Rückzug aus dem Kyoto-Protokoll über die globale Erderwärmung gelten, das Nationale Missile Defense Projekt, das eine Verletzung der ABM-Verträge mit Russland implizierte und die Ablehnung der Konvention zur Vernichtung biologischer und toxischer Waffen zu Gunsten einer unilateralen Durchsetzung des Waffenverbots durch die USA (Mahajan 2002: 22).

\section{Die Intensivierung und Legitimierung des Imperialismus: US-Außenpolitik nach dem 11. September}

Die katastrophalen Vorfälle vom 11. September dienten nicht nur dazu, die militärische Expansion und den amerikanischen Imperialismus unter dem Etikett „Krieg gegen den Terrorismus“ zu rechtfertigen, sondern auch direktere und repressivere Formen der Intervention in „strategische“ Bereiche des Südens zu legitimieren. Oberflächlich betrachtet wurde der amerikanische „Krieg gegen den Terrorismus" - sowohl in den USA als auch international - durch die Konstruktion eines amerikanischen Lieblingsmythos legitimiert: die Gleichsetzung des universell Guten mit amerikanischen Werten. Kagan bringt diese Logik auf den Punkt:

„When Americans sought legitimacy for their actions abroad, they sought it not from supranational institutions but from their own principles. That is why it was always so easy for so many Americans to believe, as so many still believe today, that by advancing their own interests they advance the interests of humanity" (Kagan 2003: 88). Diese Vorstellung drückt sich auch unmittelbar in Bushs National Security Strategy (NSS) aus (The Economist 2002b).

Unter dem Einfluss der „I am American“ Anzeigenkampagnen stieg die Zustimmung zum Präsidenten zeitweise auf erstaunliche $82 \%$ und auch die Konsumentenausgaben nahmen wieder zu. Darüber hinaus jedoch war es auf einer tieferen Ebene die Konstruktion des Mythos vom amerikanischen Internationalismus und den feindlichen Anderen, der die Intensivierung repressiver Politikformen zu legitimieren half - sowohl im eigenen Land als auch in den strategischen Arenen des Südens, namentlich: in den failed states. Im Zuge des von Präsident Bush vorgeschlagenen Haushalts 2004, der gegenüber der bereits hohen Verschuldung des Landes völlig ignorant ist, wird diese Logik auf 
die Spitze getrieben: die „unausweichliche Schlussfolgerung“ sei, dass „the federal government must restrain the growth in any spending not directly associated with the physical security of the nation" (Office of Management and Budget 2003). Es fällt auf, dass der Bundeshaushalt viel stärker an der militärischen Dimensionen von Unsicherheit ausgerichtet ist als an der Bekämpfung sozialer und ökonomischer Unsicherheiten (Financial Times 2003a). Damit wurde auch ein weiterer Schritt zur Einschränkung des US-amerikanischen Wohlfahrtssystems gemacht, obwohl es schon jetzt im Vergleich mit anderen entwickelten Industrienationen eines der regressivsten ist - sowohl was die (mangelnde) materielle Zuwendung für sozial schwache Bevölkerungsschichten angeht, als auch im Hinblick auf den strafenden und überwachenden Charakter des Systems (Platt 2003).

Ein weiterer Aspekt der NSS betrifft die Vertiefung und Ausweitung staatlicher Repression in den USA durch die Rücknahme von Bürgerrechten. Dies zeigt sich nicht nur in der Art und Weise, wie die US-Regierung versucht, das Sicherheit-Unsicherheits Paradoxon im eigenen Land zu bewältigen, sondern auch im außenpolitischen Umgang mit den Ländern des Südens. Ein ausgezeichnetes Beispiel für den Zusammenhang zwischen innen- und außenpolitischen Formen repressiver Politik bilden der Patriot Act und eine Reihe spezifischer Indikatoren des MCA, wie etwa die Implementierung ziviler und politischer Rechte. ${ }^{13}$ In Anbetracht dessen, dass Bürgerrechte innerhalb der USA zunehmend eingeschränkt werden, fragt man sich, wie die Forderung der Vereinigten Staaten, diese Rechte in den Ländern des Südens auszudehnen, eigentlich zu verstehen ist. Der Patriot Act, der im Oktober 2001 durch den amerikanischen Kongress ging, wurde öffentlich mit der Notwendigkeit begründet, angemessene Maßnahmen zur Bekämpfung des Terrorismus ergreifen zu müssen und Amerika dabei zu einen und zu stärken. Die Mittel zur Erreichung dieses Zieles bestehen in einer bislang beispiellosen Rücknahme von Bürgerrechten und der massiven Zunahme der Überwachung und Einkerkerung sowohl von Migranten als auch von US-Bürgern (Rampton/Stauber 2003).

\section{Das MCA: Eine Strategie zur Bewältigung des Sicherheit-Unsicherheits Paradoxon}

\section{Ausgeschlossene Staaten und die National Security Strategy (NSS)}

Gemäß der NSS stellen failed states eine direkte Bedrohung für die nationale Sicherheit der USA dar. An dieser Stelle ist es hilfreich den Begriff der failed states näher zu betrachten. Gemeinhin kann kaum davon die Rede sein, dass es einen Konsens über die Bedeutung dieser Bezeichnung gibt. Von der US-

13 Diese beiden Indikatoren werden vom Freedom House definiert und quantitativ gemessen. Für weitere Informationen siehe Freedom House (2003). 
Regierung werden failed states, wie etwa Nigeria, Afghanistan, Sierra Leone, Iran, Bosnien und Somalia definiert als „countries in which the central government does not exert effective control over, nor is it able to deliver vital services to, significant parts of its own territory due to conflict, ineffective governance, or state collapse“ (Rice 2003). Folgt man dieser Logik, dann stellen failed states nicht nur Operationsbasen und sichere Häfen für den internationalen Terrorismus dar. Angesichts ihrer großen „Entwicklungsdefizite“ (z.B. betreffend ökonomischer Freiheit für das Kapital und der Geltung bürgerlichen Rechts), gäbe es in diesen Staaten auch ein großes Potential an Unzufriedenheit und Gewalt, das auf andere Länder übergreifen könne (White House 2002). Repressive und kontrollierende Politiken gegenüber den betreffenden Länder beziehen ihre Legitimation aus der Position, dass alle Staaten das Recht hätten, durch ihre Politik gegenüber den failed states dem Terrorismus vorzubeugen, durch den sie selbst ansonsten geschädigt würden. ${ }^{14} \mathrm{Um}$ das MCA besser $\mathrm{zu}$ verstehen ist es hilfreich sich zu fragen, was die US-Regierung eigentlich als den Hauptursache für die Entstehung der „ausgeschlossenen Staaten“ ansieht. ${ }^{15}$ Obwohl die US-Regierung keinen einfachen Zusammenhang zwischen Armut und Terrorismus herstellen will, stellt sie doch stark darauf ab, dass ein hohes $\mathrm{Maß}$ an Armut auch ein größeres terroristisches Potential mit sich bringt. Die NSS stellt klar, „the events of September 11, 2001, taught us that weak states, like Afghanistan, can pose as great a danger to our national interests as strong states. Poverty does not make poor people into terrorists and murderers. Yet poverty, weak institutions, and corruption can make weak states vulnerable to terrorist networks and drug cartels within their borders" (Daalder et. al. 2002). Sollen zukünftige terroristische Aggressionen gegen Amerika verhindert werden, so muss die US-Regierung in dieser Logik ein höheres Maß an Kontrolle über die innenpolitischen Prozesse in den betreffenden Ländern gewinnen. Die US-Hegemonie in der globalen politischen Ökonomie soll dadurch gesichert werden, dass die failed states marktorientierte Politiken implementieren, sich der Globalisierung öffnen und so die Armut im Land überwinden. In diesem Kontext gibt es aktuell zwei Hauptstrategien, die ärmsten Regionen der Welt in die „goldene Zwangsjacke“, von der oben schon die Rede war, zu stecken: die eine besteht darin, dass die International Development Association (IDA) der Weltbank die Kredite der betreffenden Länder in Fördergelder umwandelt, deren Vergabe wie schon erwähnt an messbare Kriterien gebunden wird; die andere Strategie ist das MCA.

14 Natürlich wird von der US-Regierung nicht thematisiert, dass die Bedeutung des Begriffs „failed states" in hohem Maße subjektiv und interessengeleitet ist. Eine kritische Auseinandersetzung findet sich bei Martin Khor (2002).

15 Wenn es um die Frage geht, warum diese Staaten heute daran scheitern, den Kapitalinteressen gerecht zu werden, spielen die Jahrhunderte kolonialer und imperialistischer Ausbeutung und Unterdrückung im offiziellen Diskurs selbstverständlich keine Rolle. 


\section{Die Intensivierung neoliberaler Disziplin durch Entwicklungshilfe}

Um sicher zu stellen, dass sich die ärmsten Länder den neoliberalen Prinzipien anpassen, setzt die Bush-Regierung vor allem auf unilaterale Lösungen, die jedoch auf der Grundlage eines multilateralen Fonds, wie etwa der IDA, umgesetzt werden. Die IDA ist eine Schlüsselinstitution innerhalb der Weltbankgruppe (Pincus/Winters 2002). Sie wurde 1960 geschaffen, um die ärmsten Länder der Welt bei der Armutsbekämpfung zu unterstützen. Dazu vergibt sie zinslose Kredite mit einer Laufzeit von 35-40 Jahren und einem Tilgungsaufschub von zehn Jahren. ${ }^{16}$ Um eine maximale entwicklungspolitische Kontrolle zu erreichen, hat die US-Regierung darauf insistiert, dass die IDA ihre Kredite in Fördergelder (grants) umwandelt. Das US-Finanzministerium erklärte dazu:

„Der neue Vorschlag, den wir der Weltbank gemacht haben, besteht darin, die Beiträge der die IDA finanzierenden Länder an messbare Resultate zu knüpfen. Fördergelder können viel effektiver an eine bestimmte Erfolge gebunden werden als Langzeitkredite. Man muss eine Leistung erbringen oder man bekommt die Fördergelder nicht. (...) Die Vereinigten Staaten haben ihre Beiträge an die IDA in den 1990ern reduziert. Wir beabsichtigen diesen Trend umzukehren. Wir wollen unsere IDA-Beiträge erhöhen, aber in unseren Augen ist es essenziell, dies in einer Art und Weise zu tun, durch welche die Beiträge eng an Resultate gebunden werden." (Taylor 2002)

Das Konzept der leistungsabhängigen Fördergelder ist keine neue Idee. Der Vorschlag, traditionelle Kredite durch Fördergelder zu ersetzen, fand in neokonservativen Kreisen enthusiastische Unterstützung. Die außerordentlich einflussreichen amerikanischen think tanks, wie etwa die Heritage Foundation oder die International Financial Institution Advisory Commission, auch bekannt als Meltzer Commission, heißen das Projekt ausdrücklich gut (Schaefer 2001). Für Adam Lerrick und Allan H. Meltzer, beide Befürworter von Fördergeldern, stellen die leistungsabhängigen Fördergelder eine viel effektivere Form der Kontrolle über Staaten und Märkte im Süden dar als traditionelle Kredite. Fördergelder können zum einen an konkrete Projekte geknüpft und diese Projekte dann unter Wettbewerbsbedingungen ausgeschrieben und durchgeführt werden (Zugang haben dann auch ausländische Marktteilnehmer) und die Projektfinanzierung kann außerdem auf die Weltbank und das Empfängerland verteilt werden. Zum anderen würde das Konzept der Fördergelder eine Überprüfung der Ergebnisse auf der Grundlage quantifizierbarer Kriterien erlauben. Diese sollten auf die Verbesserung der Lebensbedingungen und damit zusammenhängend auf wirtschaftliches Wachstum abzielen: vor allem betrifft dies die Bereiche Gesundheit und Wasserversorgung, aber auch die Anzahl der geimpften Babies, die Verbesserung der Alphabetisierungsrate, usw. (Lerrick/Meltzer 2002). Das Konzept der Vergabe leistungsabhängiger Fördergelder durch die IDA stößt jedoch nicht bei allen Regierungen auf Zustimmung: zahlreiche G7-Staaten stehen diesem Vorschlag kritisch gegenüber (San-

16 Gegenüber der IDA agieren die Vereinigten Staaten bereits seit den frühen 1980er Jahren zunehmend unilateral und preemptiv, siehe Kapur (2002). 
ford 2002). Die USA versuchen daher das Konzept offensiv und unilateral zu implementieren.

2002 stellte die US-Regierung der IDA zusätzliche Finanzmittel in Aussicht, wenn diese ihre Arbeit auf der Grundlage messbarer Kriterien aufnehmen und diagnostische Einschätzungen über eine Reihe von Schlüsselländer liefern würde. Gemäß US-Finanzminister John Snow „for the first time IDA donors are able to link a portion of their contributions to the achievement of results. The U.S. committed to increase its budgetary request by 18 per cent for IDA. Of the $\$ 2.85$ billion U.S contribution to IDA, $\$ 300$ million is contingent on improving results in a concrete and measurable way." Vom Erfolg der IDA überzeugt, kündigte Snow im April 2003 dann an, sich für die Bereitstellung zusätzlicher 100 Millionen USD einzusetzen (US-Treasury Department 2003).

\section{MCA: Das neue Programm für eine globale Entwicklungspolitik}

Am 8. September 2000 verpflichteten sich die Staatschefs der Welt die Armut in ihren Ländern bis zum Jahr 2015 zu reduzieren. Um dieses Ziel der Millenium Declaration zu erreichen, lud UN-Sekretär Kofi Annan im März 2002 zu einer Financing for Development Konferenz ins mexikanische Monterrey ein. Hier kündigte Präsident Bush seine Absicht an, die US-Auslandshilfe für arme Länder über einen Zeitraum von drei Jahren hinweg massiv aufzustocken. 5 Mrd. Dollar jährlich sollten, so Bush, der US-amerikanischen Entwicklungshilfe (ODA) zusätzlich zur Verfügung gestellt werden: 1,7 Mrd. Dollar im Haushaltsjahr 2004, 3,3 Mrd. Dollar 2005 und 5 Mrd. Dollar jährlich ab 2006 (US-Agency for International Development 2002). Der Kongress reduzierte den von Bush vorgeschlagenen Haushalt um 1,8 Mrd. Dollar. Damit war dieser noch um 6\% höher als der für das laufende Finanzjahr bewilligte Betrag (US-Department of State 2003). Jedoch ändert die vom Kongress beschlossene Reduzierung der Haushaltsmittel für das MCA nichts an dessen Rolle als Trendsetter für die Entwicklungspolitik. So werden die Fonds des MCA nicht etwa von der USAID sondern von der Millennium Challenge Corporation (MCC) verwaltet, obwohl die USAID ein ,Schlüsselpartner" des MCA sein soll und viele MCA-Programme implementieren soll. Dabei versucht das Weiße Haus die MCC als unabhängige Agentur zu etablieren, deren Spitze vom Präsidenten der Vereinigten Staaten eingesetzt wird. Dagegen würde der Kongress die MCC gern unter der Obhut des Außenministeriums arbeiten lassen und somit einer stärkeren Kontrolle unterwerfen. Im Gegensatz zur traditionellen Form der Entwicklungshilfe, zielt das Programm mit seinem Profil der leistungsabhängigen Fördergelder darauf ab, Politikimplementierungen nach messbaren Resultaten zu belohnen und konkrete Handlungspläne zu entwerfen, die sicher stellen, dass die von der NSS gesteckten Ziele auch erreicht werden. Mit den Worten von Präsident George W. Bush. 
„Länder, die nach diesen drei weit gefassten Standards handeln - gerecht regieren, zum Wohl der eigenen Bevölkerung investieren, und die ökonomische Freiheit fördern - werden zukünftig von Amerika mehr Hilfe erhalten. Und, wichtiger noch, sie werden diese Hilfe nicht mehr lange brauchen, denn Nationen mit soliden Gesetzen und politischen Programmen werden mehr Auslandsinvestitionen anziehen. Sie werden größere Handelseinkommen erwirtschaften. Und sie werden merken, dass dieses Kapital effektiver und produktiver investiert wird, um mehr Jobs für die Menschen zu schaffen. Ich fordere andere Nationen und entwicklungspolitische Banken deshalb auf, dieses Konzept ebenfalls anzunehmen“ (Bush 2002).

Die MCA soll für diejenigen Länder Hilfe bereit stellen, die erfolgreich gezeigt haben, dass sie die 16 erforderlichen Kriterien erfüllen und damit drei zentralen Ansprüchen gerecht werden: (1) gerecht regieren; (2) in die Bevölkerung investieren; und (3) wirtschaftliche Freiheit garantieren (siehe Tabelle). Zwar drücken sich in diesen drei Bedingungen die zentralen Elemente des offiziellen entwicklungspolitischen Diskurses aus, z.B. der Imperativ des ökonomischen Wachstums, freier Kapitalverkehr, ein solides makroökonomisches Fundament, Good governance und demokratische Werte. Gleichzeitig wird aber die Idee des empowering durch den preemptiven Charakter des MCA überschattet. Das weiter unten aufgeführte Zitat des US-State Departments illustriert, dass das MCA den Diskurs des empowering development, etwa im Hinblick auf die Begriffe Eigentumsrechte und soziale Inklusion (Partnerschaft, Partizipation) teilt, während es aber gleichzeitig die failed states diszipliniert und kontrolliert, um sie den Marktprinzipien zu unterwerfen. Die entwicklungspolitische Sorge um die Befähigung (empowerment) der betroffenen Menschen stößt also schnell an Grenzen, wenn es darum geht, dass die politischen Reformen entlang der 16 unten aufgeführten Kriterien im Partnerland verankert werden müssen. Angesichts der Ineffizienz der Strategie der Konditionalität, wie sie vom IWF und der Weltbank angewendet wurde, betonen die Architekten des MCA die Notwendigkeit, in den betreffenden Ländern einen starken Einfluss auf innenpolitische Transformationsprozesse zu nehmen. Wie das unten folgende Zitat verdeutlicht, werden die Reformen dazu nach dem klassischen ,top-down' Verfahren implementiert und mit Formen einer intensivierten Kontrolle durch die USA kombiniert.

Im Gegensatz zum Multilateralismus, wie er sich in Clintons engagement and enlargement Strategie ausdrückte oder auch im Konzept des empowering development zeigt, zeichnen sich diejenigen Institutionen, die an den Evaluierungsprozessen auf der Grundlage der 16 Kriterien beteiligt sind, entweder durch ihre Nähe zu neo-konservativen amerikanischen Organisationen wie der Heritage Foundation und dem Freedom House aus oder sie gehören zu den von den USA dominierten internationalen Finanzinstitutionen. Jedes Urteil auf der Grundlage der oben aufgeführten Kriterien wird somit aus einer rein 


\section{Berechtigungskriterien für Fördergelder des MCA}

\begin{tabular}{|l|l|}
\hline INDIKATOR & AGENTUR \\
\hline I. „Gerecht Regieren“ & \\
\hline 1. Korruptionskontrolle & World Bank Institute \\
\hline 2. Rechtsstaatlichkeit & World Bank Institute \\
\hline 3. Politische Partizipation und Verantwortlichkeit & World Bank Institute \\
\hline 4. Effektives Regierungshandeln & World Bank Institute \\
\hline 5. Bürgerrechte & Freedom House \\
\hline 6. Politische Rechte & Freedom House \\
\hline & \\
\hline II. Investitionen in die Bevölkerung & \\
\hline 7. Anzahl der Impfungen & WHO/World Bank \\
\hline 8. Grundschulbildung: Abschlussquote & World Bank \\
\hline $\begin{array}{l}\text { 9. Grundschulbildung: } \\
\text { offentliche Ausgaben gemessen am BIP }\end{array}$ & World Bank \\
\hline $\begin{array}{l}\text { 10. Gesundheitswesen: } \\
\text { öffentliche Ausgaben gemessen am BIP }\end{array}$ & World Bank \\
\hline & \\
\hline III. Wirtschaftliche Freiheit & \\
\hline 11. Kreditwürdigkeit des Landes & Institutional Investor \\
\hline 12. Inflation & IMF \\
\hline 13. Regulierungsqualität & World Bank Institute \\
\hline 14. Haushaltsdefizit gemessen am BIP & IMF/World Bank \\
\hline 15. Handelspolitik & Heritage Foundation ${ }^{19}$ \\
\hline 16. Dauer einer Gewerbegenehmigung & World Bank \\
\hline
\end{tabular}

Quelle: Fact Sheet: Millennium Challenge Account, 25. November, 2002, http://www.cgdec.org. In: Radelet $(2003,175)$.

amerikanischen Perspektive gefällt, zum Beispiel im Hinblick darauf, wann von der Durchsetzung politischer Rechte und Bürgerrechte gesprochen werden kann. Weder die NSS noch das MCA geben eine klare Auskunft darüber, wie die 16 Kriterien inhaltlich gefüllt werden sollen, was den Ermessensspielraum der beteiligten Institutionen erheblich ausdehnt. Es dominieren kulturelle Vorurteile auf der Basis westlich-christlicher Werte und willkürliche Vorstel-

17 Das World Bank Institute (WBI) gehört zur Weltbankgruppe und schafft „learning opportunities for countries, World Bank staff and clients, and people committed to poverty reduction and sustainable development. WBI's work program includes training, policy consultations, and the creation and support of knowledge networks related to international economic and social development" (siehe http://www.worldbank.org/wbi/).

18 Das Freedom House ist „a non-profit, non-partisan organization working to advance worldwide expansion of political and economic freedom." Das Freedom House wurde etwa zur gleichen Zeit wie die Bretton Woods Institutionen gegründet und wird unter anderem von der Soros Foundation, USAID und der Ford Foundation finanziert. Weitere Informationen unter www.freedomhouse.org

19 Die 1973 gegründete Heritage Foundation ist ein wichtiger think tank, ,whose mission is to formulate and promote conservative public policies based on the principles of free enterprise, limited government, individual freedom, traditional American values, and a strong national defense" (http://www.heritage.org). 
lungen davon, wie die jeweiligen Kriterien inhaltlich definiert werden sollen. Angesichts des zunehmend repressiven Klimas in den USA, wie es sich etwa im US-Patriot Act wiederspiegelt, ist fraglich, was überhaupt unter dem Begriff der Bürgerrechte verstanden wird. Letztendlich werden hier stark interessengeleitete politische Begriffe produziert, die dann in der Öffentlichkeit als völlig selbstverständlich gelten (Said 1979). Das folgende Zitat verdeutlicht, inwieweit in der Zusammenarbeit zwischen der Heritage Foundation, einem wichtigen Akteur in der finanzpolitischen Arena, und dem Wall Street Journal, nicht nur allgemeine Annahmen über die Bedeutung wirtschaftlicher Freiheit und der Geltung bürgerlichen Rechts in den Ländern des Südens reproduziert werden, sondern dass es hier auch um handfeste Disziplinierungsprozesse im Dienste von Kapitalinteressen geht:

„Der Index of Economic Freedom wird für 161 Länder nach zehn Faktoren ermittelt, wobei für jeden Faktor Punkte zwischen 1 (bestes Ergebnis) und 5 (schlechtestes Ergebnis) vergeben werden. Die Faktoren sind: Handelspolitik, Staatsverschuldung, staatliche Interventionen in die Wirtschaft, Geldpolitik, Kapitalflüsse und Auslandsinvestitionen, Banken- und Finanzgeschäfte, Löhne und Preise, Eigentumsrechte, Regulierungen und Aktivitäten auf dem Schwarzmarkt. Die in den einzelnen Faktoren erreichten Punkte werden gemittelt, um so zu einem Gesamtergebnis zu gelangen, dass das Maß an wirtschaftlicher Freiheit beschreibt. Auf der Grundlage des Gesamtergebnisses werden die Ländern dann als ,frei', ,überwiegend frei', ,überwiegend unfrei’ oder ,unterdrückt' eingestuft“ (Schaefer 2003: 3).

Zum einen wird nun klar wie das MCA operieren wird. Zum anderen zeigt sich, dass die goldene Zwangsjacke alles andere als ein rein marktförmig vermitteltes Phänomen ist. Vielmehr handelt es sich um eine politische Strategie, die partikularen Interessen dient. Eine schlechte Bewertung auf dem Index of Economic Freedom der Heritage Foundation, der jährlich durch das Wall Street Journal publiziert wird, signalisiert ein höheres Investitionsrisiko für transnationale Kapitale. Für die betroffenen Länder hat dies Kapitalflucht oder einen Rückgang der Investitionen zur Folge. Dabei sind die wirtschaftspolitischen Annahmen, die dem Bewertungsverfahren zu Grunde liegen, höchst subjektiv und keinesfalls unumstritten. Die Korrelation zwischen wirtschaftlicher Freiheit, Wirtschaftswachstum und Demokratie ist jedenfalls alles andere als erwiesen. Historisch betrachtet war es das autoritäre Pinochet-Regime in Chile, welches in den 1980er Jahren für viele Länder des Südens zum Vorreiter des neoliberalen Modells wurde. Auch in Ostasien wurden die großen wirtschaftlichen Erfolge nicht von demokratischen sondern von autoritären Regimen erreicht. Genauso basiert die Annahme, dass wirtschaftliche Freiheit historisch und kulturell bedingten Bürgerkriegen, Unterdrückung und Leid in vielen failed states ein Ende bereiten würde, auf deformierten eurozentristischen Idealen. Diese rechtfertigen auch zunehmend physischen und ökonomischen Zwang, der durch das Zurückhalten privater Investitionen und neuerdings auch öffentlicher Hilfsmittel in den betreffenden Staaten erzeugt wird. In dieser Hinsicht ist das MCA kein neutrales entwicklungspolitisches Pro- 
gramm, sondern es ist von kulturellen und ideologischen Elementen durchdrungen. Die Konstruktion des „Wir“ und der „Anderen“ löst die Rhetorik des Kalten Krieges ab (die ständigen Beschuldigungen der westlichen Regierungen und Medien gegenüber den kommunistischen Regimen), sie trennt entlang der Linie zwischen der „Koalition der Willigen“ und der „Achse des Bösen" und verzerrt und verschleiert die wachsenden Widersprüche, welche die neoliberale Globalisierung und der amerikanischen Imperialismus in den letzten Jahrzehnten hervorgebracht haben. Das MCA hat sich die altruistischen Ziele, die in den Millenium Development Goals formuliert werden, angeeignet, ihre Bedeutungen verkehrt und dient vor allem den politischen Zielen des amerikanischen Empire. Obwohl das neue globale Entwicklungshilfeprogramm primär auf der Grundlage von Zwang operiert, kann es sowohl in der US-amerikanischen Öffentlichkeit als auch auf internationaler Ebene auf breite Legitimität bauen. Diese wird vor allem durch einen Diskurs produziert, der „die Anderen“ als eine passive, schweigende und homogene Einheit beschreibt, welche sich der neoliberalen Modernisierung verweigert und daher stets eine potentielle Bedrohung für „den Westen“ bleibt. Dieser mit dem MCA eng verwobene Diskurs degradiert die Menschen in den kolonisierten Kulturen zu machtlosen Objekten. Edward Said weist auf ein zentrales Element in der Durchsetzung dieser Kultur der Machtlosigkeit hin: das in den ausgeschlossenen Staaten produzierte Wissen wird von den Massenmedien, von bilateralen Partnern, privaten Kreditgebern und Investoren schlicht und ergreifend als illegitim erachtet, als nicht-Wissen, als Nonsens (Said 1979). Das Resultat der Kultur des „machtlosen Anderen“ im offiziellen Entwicklungsdiskurs, insbesondere im MCA, ist die allgemeine Annahme, dass es nur einen Weg der „Entwicklung“ gibt: die Akzeptanz der neoliberalen Globalisierung und der westlichen Demokratie.

\section{Fazit}

Das MCA steht für eine offensive, repressive und vor allem US-dominierte Form der Entwicklungspolitik, deren Inhalte sich aber von denen vorangegangener entwicklungspolitischer Programme nicht substantiell unterscheiden. Aus einer historisch-materialistischen Perspektive zeigt sich, dass die Genese und die innere Natur des MCA als Momente der Restrukturierung des amerikanischen Imperialismus im widersprüchlichen Kontext neoliberaler Globalisierungsprozesse verstanden werden müssen. Mit dem Versuch, das MCA zu dekonstruieren, sollte hier über die allgemeine Annahme hinausgegangen werden, dass das MCA ein unmittelbares Resultat der tragischen Vorfälle vom 11. September darstellt. Statt dessen wurde hier eine Auseinandersetzung mit dem Inhalt des MCA und der zunehmend offensiven und repressiven Politik des amerikanischen Empires gegenüber den Ländern des Südens geleistet. Dabei 
wurde gezeigt, dass Bushs preemptive Entwicklungsagenda aus dem Zusammenwirken von mindestens drei Faktoren entstanden ist - welche nicht etwa durch die Vorfälle vom 11. September 2001 verursacht wurden, sondern vielmehr in diesen gipfelten. Erstens: Insbesondere seit dem Zerplatzen der spekulativen Blasen an der Börse kann nicht nur in der USA eine Krise des Vertrauens in die neoliberale Regierungspolitik beobachtet werden. Auch in den ausgeschlossenen Staaten ist diese Vertrauenskrise gegenüber dem Neoliberalismus virulent geworden. In den meisten Drittwelt- und Schwellenländern sind die Einkommensniveaus gefallen, Armutsraten steigen und die Schere zwischen reichen und armen Ländern hat sich geöffnet. Zweitens: Ein Großteil der westlichen Investitionen fließt in die emerging markets. Die ärmsten Länder, welche die Zielgruppe des MCA darstellen, erhalten dagegen kaum private Investitionen und auch die multilateralen und bilateralen Hilfsleistungen für diese Länder nehmen ab. Drittens hat die US-Ökonomie, die bislang als weltweiter Wachstumsmotor fungierte, nach dem Crash vom August 2000 einen deutlichen wirtschaftlichen Abschwung erfahren. Die Arbeitslosigkeit nimmt zu und befindet sich auf dem höchsten Niveau seit 1994, das Haushalts- und Handelsdefizit wächst schnell, die Konsumenten- und Unternehmensschulden weisen auf eine Schwächung der US-Ökonomie hin. Im Gegensatz zu dem, was die Rhetorik des amerikanischen „Krieg gegen den Terrorismus“ offiziell verlautbart, richtet sich die Sicherheitspolitik der USA mehr und mehr an der Notwendigkeit aus, konstant und zunehmend repressiv in die Staaten und Märkte des Südens zu intervenieren, um die eigenen Interessen dort zu schützen. Diese Politik steht in krassem Gegensatz zu der lauthals verkündeten Absicht, Demokratie und Freiheit in die Welt zu tragen. Es scheint, dass der Trend zum intensivierten ökonomischen und militärischen Zwang zur dominanten Strategie wird, um das „New American Century“ zu erreichen.

Übersetzung aus dem Englischen von Henrik Lebuhn

\section{Literatur}

Albo, Gregory (2004): The Old and New Economics of Imperialism, in: Panitch, L., Leys, C. (Hg.), Socialist Register 2004: The New Imperial Challenge, London, 88-113.

Altvater, Elmar (2002): The Growth Obsession, in: Panitch, L., Leys, C. (Hg.), Socialist Register, London.

Altvater, Emar/ Mahnkopf, Birgit (2002): Globalisierung der Unsicherheit, Münster.

Amin, S. (1999): For Progressive and Democratic New World Order, in: Adams, Francis et. al. (Hg.), Globalization and the Dilemmas of the State in the South, London, 17-32.

Brodie, Janine (2003): Globalization, In/security, and the Paradoxes of the Social, in: Bakker, Isabella, Gill, Stephen (Hg.), Power, Production and Social Reproduction, London, 47-32.

Bush, G. W. (2002): The U.S.'s Millennium Challenge Account: New Paradigm For Development Assistance, in: Citizens' Network on Essential Services: News \& Notices for IMF and World Bank Watchers, Vol. 2, Nr. 8, Spring 2003, http://www.challengeglobalization.org/ $\mathrm{html} /$ news_notices/spring2003/INTRO.

Clinton, W. J. (1998): Remarks by the President to Opening Ceremony of the 1998 International Monetary Fund, World Bank Annual Meeting. 
Commission on Global Governance (1995): Report of the Commission on Global Governance: Our Global Neighbourhood, Oxford.

Daalder, Ivo H. et al. (2002): The Bush National Security Strategy: An Evaluation, The Brookings Institution, Policy Brief No. 109, Washington, D.C.

Dodge, Toby (2002): Iraq and the Bush Doctrine, The Observer, March 24, 2002, http://www.observer.co.uk/worldview/story/0,11581,672584,00.html.

Feulner, Edwin J. (1998): The IMF Needs Real Reforms, Not More Money, Heritage Foundation Backgrounder No. 1175, May 9, 1998.

Financial, Times (2003a): Doubts arise over scope for US reforms, in: Financial Times, 13.Feb.

- (2003b): Unemployment up to 6.1 per cent in May. in: Financial Times, 6. Juni.

Fine, Ben (2001): Making the post-Washington consensus, in: Fine, Ben et al (Hg.), Development Policy in the Twenty-first Century: Beyond the post-Washington consensus, London, 131-154.

Freedom, House. (2003). Freedom in the World: Country and Territory Report. Freedom House, http://www.freedomhouse.org/research/freeworld/2003/countries.htm.

Friedman, Thomas (1999): The Lexus and the Olive Tree, London.

Gill, Stephen, David Law (1993): Global Hegemony and the Structural Power of Capital, in: ders. (Hg.): Gramsci, Historical Materialism and International Relations, Cambridge, 93-124.

Graham, Carol/ Masson, Paul. (2002): The IMF's Dilemma in Argentina: Time for a New Approach to Lending? The Brookings Institution, Policy Brief \#111. Washington, D.C.

Harvey, David (2004): The 'New' Imperialism: Accumulation by Dispossession, in: Panitch, L., Leys, C. (Hg.), Socialist Register 2004: The New Imperial Challenge, London, 63-87.

Hoffman, Stanley (2002): Clash of Globalizations, in: Foreign Affairs, 81.Jg., H.4, Juli/August.

IMF (2003): Poverty Reduction Strategy Papers, 26. June, http://www.imf.org/external/np/prsp/prsp.asp

Johnson/ Schaefer. (1997): No New Funding for the IMF. Heritage Foundation Backgrounder Update No. 287, 23. September.

Kagan, Robert (2003): Of Paradise and Power: America and Europe in the New World Order, New York.

Kaldor, M. (1999): New and Old Wars: Organised Violence in a Global Era, Stanford.

Kapur, Devesh (2002): The Changing Anatomy of Governance of the World Bank, in: Pincus, J.,Winters, J.A. (Hg.), Reinventing the World Bank, Ithaca, 54-75.

Khor, Martin (2002): Failed States' theory can cause global anarchy, Third World Network, March 4, 2002, http://www.twnside.org.sg/title/et0125.htm.

Kotz, David (2003): Neoliberalism and the U.S. Economic Expansion of the 90s, in: Monthly Review, Vol. 54, Nr. 11, April 2003.

Lerrick, A./ Meltzer, A. H. (2002.): Grants: A Better Way to Deliver Aid, Quarterly International Economics Report. Gailliot Center for Public Policy, http://www.house.gov/jec/imf/grant.pdf

Litwak, Robert S. (2000): Rogue States and US Foreign Policy: Containment after the Cold War. The Woodrow Wilson International Centre for Scholars, Washington, D.C.

Mahajan, Rahul (2002): The New Crusade: America's War on Terrorism, New York.

Monthly Review (2003): Editorial: What Recovery?, in: Monthly Review, Vol. 54, Nr. 11, April.

Morrissey, M./ Baker, D. (2003): When Rivers Flow Upstream: International Capital Movements in the Era of Globalization, Issue Brief, 22. März, Centre for Economic and Policy Research, Washington, D.C, 1-21.

Ocampo, J. A. (2000): A Broad Agenda for International Financial Reform, in: Ocampo, J.A. et. al. (Hg.), Financial globalization and the emerging economies, Santiago (Chile): United Nations Economic Commission for Latin American and the Caribbean

Office of Management and Budget (2003): Charting a Course for the Federal Budget, The White House, 15. Juni, Washington, D.C., http://www.whitehouse.gov/omb/budget/fy2004/charting.html

Panitch, Leo/ Gindin, Sam (2004): Global Capitalism and American Empire, in: Panitch, L., Leys, C. (Hg.), Socialist Register 2004: The New Imperial Challenge, London, 1-42.

Pincus, Jonathan R./ Winters, Jeffrey A. (2002): Reinventing the World Bank, in: Pincus, J.,Winters, J. (Hg.), Reinventing the World Bank, Ithaca.

Platt, Tony (2003): The State of Welfare: United States 2003, in: Monthly Review, Vol. 55, Nr. 5, 13-27.

Radelet, Steve (2003): Will the Millennium Challenge Account be Different? The Washington Quarterly, Spring 2003, Vol. 26 (2).

Rampton, Sheldon/ Stauber, John. (2003): Trading on Fear, in: The Guardian, 12. Juli. 
Rice, Susan E. (2003). The New National Security Strategy: Focus on Failed States, The Brookings Institution, Policy Brief No. 116, Februar 2003, Washington, D.C.

Said, Edward (1979): Orientalism. Vintage Books, New York.

Sanford, J.E. (2002): World Bank: IDA Grants or IDA Loans. Bank Information Centre, 8.Feb., Washington, D.C, http://www.bicusa.org/usgovtoversight/Sanford\%20_IDA_loans_or_grants.pdf

Schaefer, Brett D. (2001). Real Help for Poor Nations: President Bush's World Bank Grant Proposal, The Heritage Foundation, Backgrounder \#1466, Washington, D.C., http://www.heritage.org/ Research/TradeandForeignAid/BG1466.cfm

- (2003): Promoting Growth and Prosperity in the Developing World through Economic Freedom, in: US-Department of State: Economic Perspectives: Millennium Challenge Account: A New Compact for Global Development, March 2003, Washington, D.C., http://usinfo.state.gov/ journals/ites/0303/ijee/schaefer.htm

Shapiro, Isaac (September 2000): Trends in US Development Aid and the Current Budget Debate. The Centre on Budget and Policy Priorities (Hg.), Washington, D.C. http://www.cbpp.org/ 4-25-00bud.htm

Shiller, Robert J. (2000): Irrational Exuberance, New York.

Söderberg, Susanne (im Erscheinen): Global Governance and American Empire, London.

- (2004): The Politics of the New International Financial Architecture: Reimposing Neoliberalism in the Global South, London.

Taylor, John B. (2002). Improving the Bretton Woods Financial Institutions, Citizens' Network on Essential Services: Annual Mid-Winter Strategic Issues Conference, Association for Finance and Trade, 7. Feb., Bankers, http://www.challengeglobalization.org/html/econ_lit/treasury_feb02.shtml

The Conference Board (2003): U.S. Economic Growth Will Slow in 2003; Globalization May be Stagnating, The Conference Board, 3. April, http://www.conference-board.org/economics/ press.cfm?press_ID $=2115$

The Economist (2003): Bush goes for Broke, in: The Economist, 8. Jan..

- (2002a): The O'Neill Doctrine, in: The Economist, 27. April, 12.

- (2002b): Unprecedented power, colliding ambitions, in: The Economist, 28.Sep., 27.

Thomas, Caroline (2002): Global governance and human security, in: Wilkinson, R., Hughes, S. (Hg.), Global Governance: Critical Perspectives, London.

UNDP (1999): Human Development Report 1999: Globalization with a Human Face, http://hdr.undp.org/reports/global/1999/en/

- (1994): Human Development Report, Oxford. Oxford University Press (Hg.), Oxford.

US-Agency for International Development (2002): Millennium Challenge Account Update, June 3, 2002, http://www.usaid.gov/press/releases/2002/fs_mca.html.

US-Department of State (2003): House Panel Approves \$17.1 Billion Foreign Spending Bill, 11. Juli, Washington, D.C. http://usinfo.state.gov/xarchives/display.html?p=washfile-english\&y $=2003 \& \mathrm{~m}=J u l y \& \mathrm{x}=20030711103326 \mathrm{emmoccmk} 7.867068 \mathrm{e}-02 \& \mathrm{t}=\mathrm{usinfo} /$ wf-latest.html

US Treasury Department, Office of Public Affairs of the US Treasury Department (2003): Treasury Secretary John Snow Announces Request of an Additional \$100 million for the International Development Association, Affirms Progress on Goals and Measurable Results, 13. April, Washington, D.C, http://www.ustreas.gov/press/releases/js186.htm

White House (2002): National Security Strategy, Washington D.C.

Willetts, P. (1999): Actors in Global Politics, in: Baylis, J., Smith S. (Hg.), The Globalization of World Politics: An Introduction to International Relations, Oxford.

World Bank (o.J.): Four Elements of Empowerment, PovertyNet, http://www.worldbank.org/ poverty/empowerment/whatis/incl.htm.

- (2003): Global Development Finance: Striving for Stability in Development Finance, Washington, D.C. 